\title{
Detection of maleimides and their characteristics in Neogene sediments of the Shinjo basin, Japan
}

\author{
Masaki Kozono, Shinya Nomoto, Hajime Mita and AKIRa Shimoyama* \\ Department of Chemistry, University of Tsukuba, Tsukuba 305-8571, Japan
}

(Received April 2, 2001; Accepted June 5, 2001)

\begin{abstract}
Maleimides were detected in organic solvent extracts and chromic acid oxidation products of Miocene to Pliocene sediments of the Shinjo basin and were very likely degradation products of porphyrins and/or chlorophylls. These maleimides included a series of 2-methylmaleimides with 3-n-alkyl substituents up to $\mathrm{C}_{5}$, benzomaleimide (phthalimide) and its methyl homologs, and tetrahydrobenzomaleimide. The total concentrations of free (the organic solvent extractable) and bound (the oxidatively extractable) maleimides ranged from 0.10 to 21 and from 1.5 to $75 \mathrm{nmol} \mathrm{g}^{-1}$, respectively. The most abundant compound was 2methyl-3-ethylmaleimide both in free and bound forms in most of the sediments. The concentrations of free and bound maleimides showed different depth profiles from those of the reported extractable organic compounds (EOC) and the total organic carbon (TOC). Their ratios to TOC decreased with increasing depth of the sediments in the oil-generating zone of the sedimentary sequence and were characteristically low in the high EOC/TOC region. The molar compositions of free maleimides did not show marked dependence on the sediment depth, whereas those of the bound forms did. The depth profile of the molar ratio of bound 2,3-dimethyl- to 2-methyl-3-ethylmaleimide showed a clearly increasing trend with depth, which agreed well with those of other widely-accepted maturity parameters.
\end{abstract}

\section{INTRODUCTION}

A large number of organic geochemical studies has been reported on porphyrins in sediments and crude oils, since the study by Treibs (1936). Porphyrins are derived principally from chlorophylls and are commonly present in sediments or crude oils. Many of the porphyrin studies have dealt with structural determination, ratios of structural isomers, and their complexes with metal from the viewpoint of understanding their origin (Callot et al., 1990; Ocampo et al., 1992), depositional environment (Lewan and Maynard, 1982) and diagenesis (Gallango and Cassani, 1992). On the other hand, studies on porphyrin decomposition and its products in sediments have not been commonly reported and, therefore, more studies are necessary on these matters.

Alkylated maleimides were first found explic- itly in the chromic acid oxidation products of porphyrins isolated from a crude oil by Martin et al. (1980). They detected a homologous series of 2-methylmaleimides with $n$-alkyl and branched alkyl side chains at 3-position, and discussed the origin of the alkyl groups in relation to diagenetic change of precursor chlorophylls. Barwise and Whitehead (1980) discovered benzomaleimides (phthalimides) from Boscan distillate by using the oxidative degradation method. These compounds were derived from so-called rhodoporphyrins that contain a benzopyrrole or a benzopyrroline ring. The presence of maleimides in sediments was reported initially by Grice et al. (1996), who found 2-methyl- and 2-ethylmaleimides with $n$-alkyl and branched alkyl side chains up to $\mathrm{C}_{5}$ in organic solvent extracts of Permian and Mid-Triassic marine sediments. The maleimides are evidently oxidation products of tetrapyrrole compounds in

*Corresponding author (e-mail: ashimoya@staff.chem.tsukuba.ac.jp) 
sediments, but the nature and timing of the oxidation have not been elucidated. Although maleimides can be important biomarker compounds that can be linked to chlorophylls, their kinds and concentrations over a sedimentary sequence have not hitherto been reported.

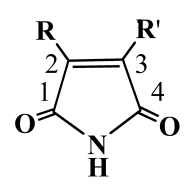

Maleimide

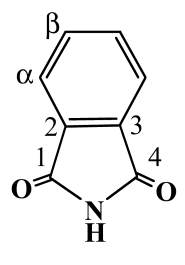

Benzomaleimide (Phthalimide)

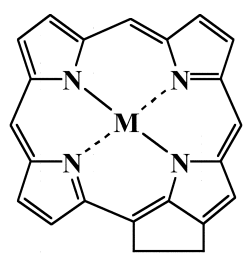

Tetrapyrrole skeleton of porphyrin
In the present investigation, we analyzed maleimides in the Neogene Shinjo sediments. The sediment samples were portions of common samples collected in an old oil field for the joint study of organic and inorganic geochemistry and some of the results have already been reported (Taguchi et al., 1986). We first extracted the sediments with organic solvents to determine extractable or "free" maleimides, and then the same samples were reextracted oxidatively with chromic acid in a similar manner to that reported by Furlong and Carpenter (1988) to determine "bound" maleimides that had been incorporated into kerogen, possibly as porphyrins or their diagenetically modified forms with pyrrole rings unchanged. This investigation provides the second example of detecting free maleimides in sediments and the first of elucidating the depth profiles of their concentrations in a stratigraphic sequence of sediments. We also evaluate the distribution patterns of free and bound maleimides over the sedimentary sequence, especially for the purpose of elucidating diagenetic effects.

\section{EXPERIMENTAL}

\section{Samples}

Sediment samples analyzed were collected at outcrops along the Oashizawa route in the Shinjo basin in 1980 . The sediments are chiefly marine shales and mudstones of the Nakawatari, Noguchi, Furukuchi and Kusanagi Formations of late to middle Miocene in stratigraphically descending order. The rock faces of the sediments and sample horizons were shown in the stratigraphic and lithologic sections of these formations by Taguchi et al. (1986). The sediment samples were numbered from 83110 to 90105 representing the top and bottom horizon, respectively, of the $1600 \mathrm{~m}$ thick sedimentary sequence.

Approximately $100 \mathrm{~g}$ each of freshly powdered samples had been prepared from hand sized specimens of the common samples. A portion of each had already been used for studies of organic compounds (Shimoyama and Matsubaya, 1985; Shimoyama et al., 1988, 1991, 2000; Mita et al., 1996; Hagiwara et al., 2000; Nomoto et al., 2000; Katsumata and Shimoyama, 2001). In this study, maleimides were analyzed in 18 samples over the sedimentary sequence.

\section{Analyses}

For each analysis, $1.0 \mathrm{~g}$ of powdered sample was extracted by sonication with $3 \mathrm{ml}$ of a mixture of dichloromethane and acetone $(9: 1, \mathrm{v} / \mathrm{v})$. After centrifugation, the supernatant was saved. The extraction and centrifugation processes were repeated four times. The extracts were combined and carefully concentrated under reduced pressure and were applied to a silica gel column $(175 \mathrm{~mm}$ $\times 10 \mathrm{~mm}$ i.d.). Elution was carried out with hexane, dichloromethane and then, a mixture of dichloromethane and acetone $(9: 1, \mathrm{v} / \mathrm{v})$. Maleimides were recovered in the final eluent. The eluent was concentrated to $50 \mu$ under a stream of nitrogen, and analyzed by a gas chromatograph combined with a mass spectrometer (GC-MS). Maleimides recovered from this fraction were named free maleimides.

The sediment samples recovered after the above extraction were oxidized with chromic acid according to the method of Furlong and Carpenter (1988), which includes the following treatments. To a suspension of the sediment sample in $10 \mathrm{ml}$ of $25 \%$ sulfuric acid (w/v) were added 
$10 \mathrm{ml}$ of $10 \%$ chromium trioxide (w/v) below $0^{\circ} \mathrm{C}$. The suspension was stirred for 2 hours below $0^{\circ} \mathrm{C}$ and then for 2 hours at room temperature. After centrifugation, the supernatant was recovered and extracted five times by shaking with $5 \mathrm{ml}$ of benzene. The benzene extracts were combined and concentrated carefully to $50 \mu$ under a nitrogen flow and analyzed by GC-MS. Maleimides obtained from the oxidative extraction of the solventextracted sediment samples were named bound maleimides.

Hexane and chromium trioxide used were of analytical grade (Wako pure chemical). Other organic solvents, such as dichloromethane, acetone and benzene, were doubly distilled before use. Water used as the solvent for chromic acid oxidation was obtained by a successive treatment of distillation and deionization. To avoid laboratory contamination, all glassware was heated at $500^{\circ} \mathrm{C}$ for 3 hours before use.

The samples were analyzed by GC-MS using a Hewlett Packard G1800A GCD system. The GC was equipped with an FFAP bonded FS-WCOT capillary column $(60 \mathrm{~m} \times 0.25 \mathrm{~mm}$ i.d.). Oven temperature was programmed to give $50^{\circ} \mathrm{C}$ for $2 \mathrm{~min}$, to increase from $50^{\circ} \mathrm{C}$ to $150^{\circ} \mathrm{C}$ at a rate of $10^{\circ} \mathrm{C}$ $\mathrm{min}^{-1}$, then from $150^{\circ} \mathrm{C}$ to $230^{\circ} \mathrm{C}$ at a rate of $3^{\circ} \mathrm{C}$ $\mathrm{min}^{-1}$, and to hold at the final temperature for 50 min. Helium was used as a carrier gas with a flow rate of $1 \mathrm{ml} \mathrm{min}^{-1}$. Electron impact $(70 \mathrm{eV})$ mass spectra were obtained by scanning from $\mathrm{m} / \mathrm{z} 45$ to 425 every $0.5 \mathrm{~s}$.

Identification of maleimides was made by comparison of retention times on the GC and of MS fragment patterns with those of standard compounds: 2-methylmaleimide, 2,3dimethylmaleimide, 2-methyl-3-ethylmaleimide, benzomaleimide (phthalimide), $\quad \beta$ methylbenzomaleimide (4-methylphthalimide), tetrahydrobenzomaleimide. These compounds were prepared in this study, except for benzomaleimide (Wako pure chemical). Abundances of maleimides were estimated by comparing peak areas on mass fragmentograms of molecular ions to those of the standard compounds.

\section{RESULTS AND DISCUSSION}

\section{Identification}

Nine mass fragmentograms of the oxidative

Table 1. Maleimides in Neogene sediments of the Shinjo basin and their major mass numbers $(\mathrm{m} / \mathrm{z}$ ) for identification

\begin{tabular}{|c|c|c|}
\hline Label* $^{*}$ & Compound & Major mass numbers $(\mathrm{m} / \mathrm{z})$ \\
\hline A & 2-Methylmaleimide & 111 \\
\hline $\mathrm{B}$ & 2,3-Dimethylmaleimide & 125 \\
\hline $\mathrm{C}$ & $\begin{array}{l}\text { 2-Methyl-3-ethylmaleimide } \\
\text { (2-Ethyl-3-methylmaleimide)** }\end{array}$ & 139 \\
\hline $\mathrm{D}$ & 2-Methyl-3-propylmaleimide & 153,125 \\
\hline $\mathrm{E}$ & $\begin{array}{l}\text { 2-Methyl-3-butylmaleimide } \\
\text { (2-Butyl-3-methylmaleimide)** }\end{array}$ & 167,125 \\
\hline $\mathrm{F}$ & 2-Methyl-3-pentylmaleimide & 181,125 \\
\hline $\mathrm{G}$ & Benzomaleimide (Phthalimide)** & 147 \\
\hline $\mathrm{H}$ & $\begin{array}{l}\text { Tetrahydrobenzomaleimide } \\
(3,4,5,6 \text {-Tetrahydrophthalimide }) * *\end{array}$ & 151 \\
\hline I & $\begin{array}{l}\alpha \text {-Methylbenzomaleimide } \\
\text { (3-Methylphthalimide)** }\end{array}$ & $161,143,133,118,90$ \\
\hline $\mathrm{J}$ & $\begin{array}{l}\beta \text {-Methylbenzomaleimide } \\
\text { (4-Methylphthalimide)** }\end{array}$ & $161,118,90$ \\
\hline
\end{tabular}

*Compounds labeled as A, B, C, G, and J are identified and quantified by using standard compounds. $\alpha$-Methylbenzomaleimide is determined by comparison of the mass spectrum with its structural isomer $\beta$-methylbenzomaleimide. Compounds labeled as $D, E$ and $F$ are identified by the mass spectra reported by Martin et al. (1980) and Barwise and Whitehead (1980).

**Named following IUPAC nomenclature. 


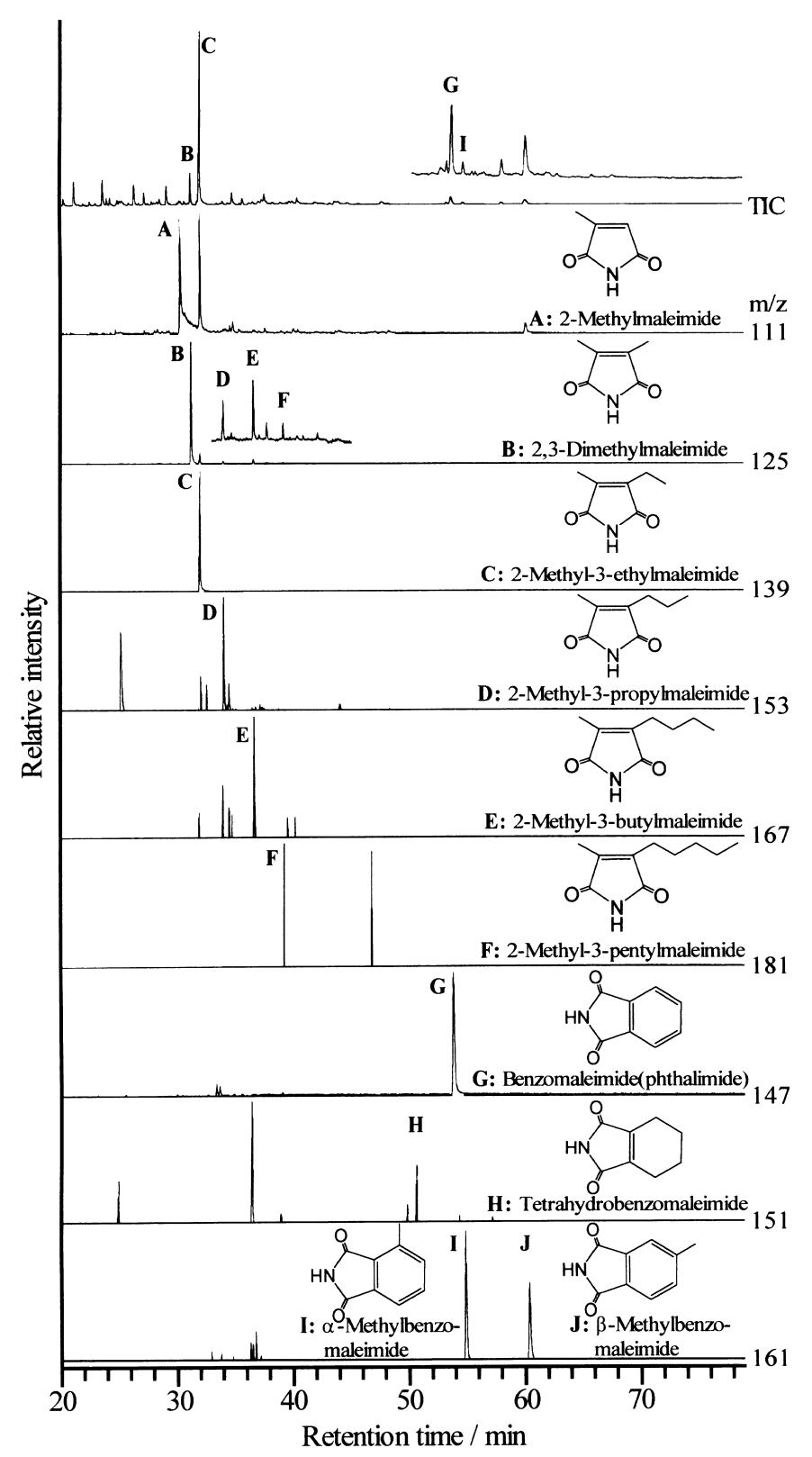

Fig. 1. Total ion chromatogram and mass fragmentograms of bound maleimides in the Shinjo sediment sample 83101 .

extract of the sample 83101 together with a total ion chromatogram are shown in Fig. 1. These mass fragmentograms show the presence of ten maleimides as listed in Table 1 . These compounds include a series of 2-methylmaleimides with $n$ alkyl substituents up to $\mathrm{C}_{5}$ as well as benzomaleimides including $\alpha$ - and $\beta$ methylbenzomaleimides, and tetrahydrobenzomaleimide. Methylmaleimides with substituents from $\mathrm{C}_{3}$ to $\mathrm{C}_{5}$ were identified by comparing their mass spectra with those reported by Barwise and Whitehead (1980), and Martin et al. (1980). 


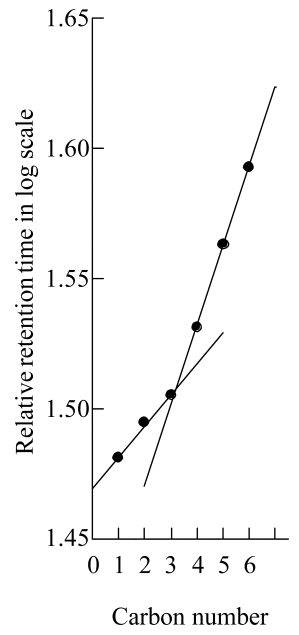

Fig. 2. Plot of logarithm of retention time vs. total carbon number of n-alkyl substituents of maleimides in the Shinjo sediments.

$\alpha$-Methylbenzomaleimide was assigned by comparing the fragmentation pattern with that of the $\beta$-methyl isomer and tentatively quantified by using the peak area/mole ratio of a standard compound of the latter isomer. As pointed out by Barwise and Whitehead (1980), and Martin et al. (1980), a relationship between carbon number of side chains on 2-methyl-3-n-alkylmaleimides and the logarithm of their retention times showed a clear discontinuity at $\mathrm{C}_{3}$ maleimide, as depicted in Fig. 2. This fact also supports our assignment of these compounds, although our GC analytical conditions were somewhat different from theirs.

These maleimides were found in both the direct extracts and the oxidative extracts from the sediments. Martin et al. (1980) detected a small amount of maleimide with no substituent in oxidation products of a crude oil only when it was converted into a trimethylsilyl derivative. However, we did not find this compound and confirmed its absence from all samples by a coinjection experiment using a standard compound. Furthermore, no evidence was obtained in support of the presence of maleimides with branched alkyl side chains.

\section{Concentrations and their depth profiles}

Table 2 gives the concentrations of free and bound maleimides. All the sediments were found to contain maleimides in free and bound forms ranging in concentration from 0.10 to 21 and from 1.5 to $75 \mathrm{nmol} \mathrm{g}^{-1}$, respectively. In almost all the sediments, the concentrations of free maleimides including benzomaleimides were about one order of magnitude smaller than those of the bound forms. Concentrations of 2-methyl-3-ethylmaleimide, the most predominant maleimide in both free and bound forms, were 11 and 68 nmol $\mathrm{g}^{-1}$, respectively. Free methylbenzomaleimides were found only in the sample 83003 in small concentrations, while the bound were present in almost all the samples. However, their concentrations were about 2 orders of magnitude smaller than those of 2-methyl-3-ethylmaleimide. The other maleimides, identified but not quantified, were even lower in concentration than methylbenzomaleimides as judged from their peak areas on the mass fragmentograms.

The ratios of the concentrations of free and bound maleimides to TOC at the 18 sediment horizons over the stratigraphic sequence are plotted in Fig. 3, along with the contents of the total organic carbon (TOC) and the extractable organic compounds (EOC) (Shimoyama and Matsubaya, 1985). The joint study on the organic and inorganic diagenesis in this sequence revealed that petroleum formation took place mainly in the Kusanagi Formation (Taguchi et al., 1986). The $T_{\max }$ value (temperature of maximum hydrocarbon generation from kerogen by Rock-Eval pyrolysis) is about $427^{\circ} \mathrm{C}$ at the upper Kusanagi Formation and this value is considered to be the threshold of intense oil generation for Japanese source rocks (Connan, 1974; Kaneko et al., 1983). The stratigraphic profile of EOC/TOC shows oil generation at the upper to middle Kusanagi Formation.

The ratio of free maleimides (Fig. 3(a)) in the Shinjo sedimentary sequence, on the whole, decreases with increasing depth, except for the top sample, contrary to the depth profiles of EOC and 


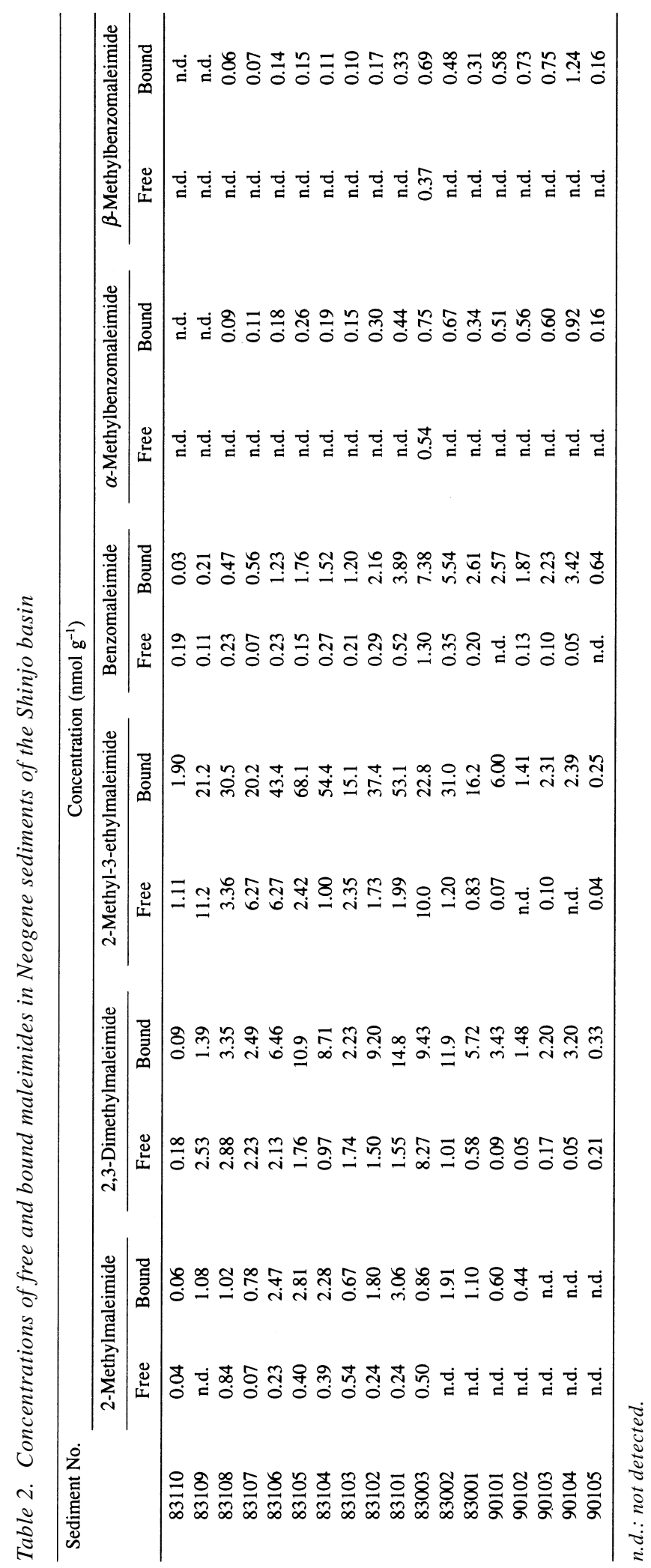


a)

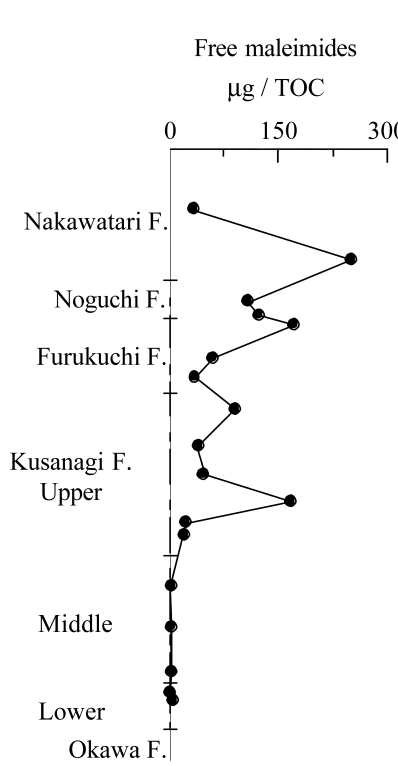

b)

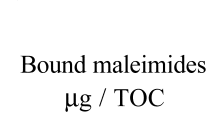

c)

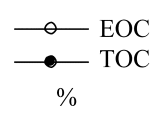

d)
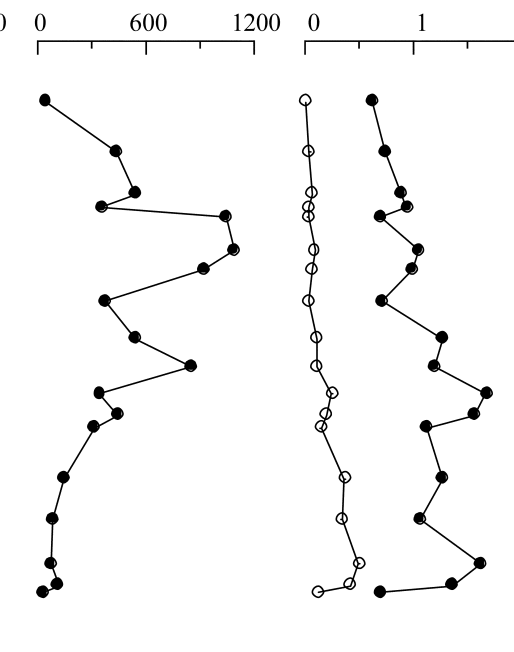

$\mathrm{EOC} / \mathrm{TOC}$

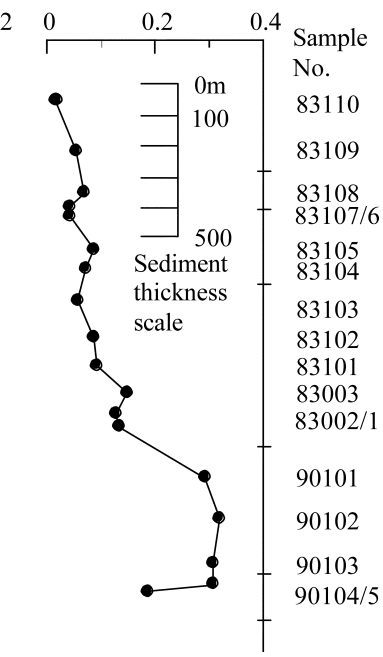

Fig. 3. Contents of free maleimides (a) and bound maleimides (b), and contents of total organic carbon (TOC) and extractable organic compounds $(E O C)(c)$, and EOC/TOC ratio $(d)$ vs. depth in the stratigraphic sequence of the Shinjo sediments. Total organic carbon and extractable organic compound data and their ratios are from Shimoyama and Matsubaya (1985).

TOC. The ratio of bound maleimides (Fig. 3(b)) shows an increasing trend with depth in the sediment region above the Kusanagi Formation, but it decreases similarly to that of free maleimides in the oil-generating zone. The ratios of both free and bound maleimides are characteristically lower in the high EOC/TOC region, where intense oil generation is inferred to have occurred. These characteristic features possibly indicate that maleimides are susceptible to diagenetic modification because of the presence of rather reactive functional groups, such as an imide group and a carbon-carbon double bond, in their structures. Another explanation for these characteristic features is a relatively small concentration of kerogen-bound porphyrins in the Kusanagi Formation, which could release only a small amount of maleimides by the chromic acid oxidation. Taguchi et al. (1970) reported that the concentration of free porphyrins was higher in the formation than above it. These porphyrins were probably released from kerogen-bound porphyrins in the formation during diagenesis. If so, a small concentration of the kerogen-bound porphyrins was left in the formation.

\section{Relation to porphyrins and chlorophylls}

The predominant maleimide, i.e., 2-methyl-3ethylmaleimide, in both free and bound forms in the Shinjo sediments is also abundant in petroleums and sediments previously reported (Furlong and Carpenter, 1988; Barakat and Yen, 1989; Grice et al., 1996, 1997; Barakat and Rullkötter, 1998). The predominance had been attributed to the fact that many chlorophylls and major sedimentary porphyrins include pyrrole or pyrroline rings with a methyl and an ethyl group, and yield 2-methyl-3-ethylmaleimide upon degradation. The presence of other maleimides with $n$-alkyl side chains up to $\mathrm{C}_{11}$ in petroleums and sediments was also reported by several investigators (Martin et al., 1980; Barwise and Whitehead, 1980). Of these, 2-methyl-, 2,3-dimethyl- and 2methyl-3-propylmaleimide have been considered 
to be derived from a source similar to that of 2methyl-3-ethylmaleimide on structural grounds and, in many cases, 2,3-dimethylmaleimide was the second most abundant, as in our case. The other alkylated maleimides have not been structurally related to the sedimentary porphyrins.

The concentrations of bound benzomaleimide (phthalimide) in the Shinjo sediments were almost comparable to those of bound 2,3-dimethylmaleimide. The relatively high concentrations of bound benzomaleimide in the sediments could indicate the presence of benzoporphyrins bound to kerogen in amounts comparable to the other porphyrin concentrations in the sediments. Furthermore, the detection of tetrahydrobenzomaleimide in the extracts suggests the presence of tetrahydrobenzoporphyrins in sediments, which in turn may be transformed to benzoporphyrins in the course of diagenesis (Barwise and Roberts, 1984; Chicarelli et al., 1987; Verne-Mismer et al., 1987; Van Berkel et al., 1989a). However, benzoporphyrins could not be correlated directly to chlorophylls, since no chlorophyll with a benzene or a cyclohexane ring fused to a tetrapyrrole skeleton is known in organisms. Nevertheless, several studies have reported the occurrence of sedimentary benzoporphyrins in relatively high abundances, i.e., up to $35 \%$ of total porphyrin content (Baker and Louda, 1986; Kaur et al., 1986; Van Berkel et al., 1989b).

Two bound $\alpha$ - and $\beta$-methylbenzomaleimides were found in almost all the sediments in concentrations comparable to those of benzomaleimide, which may imply that the methyl group has the same origin as the benzomaleimide structure. Barwise and Whitehead (1980) detected an isomer of methylbenzomaleimide as well as benzomaleimide in the oxidation product of a rhodoporphyrin fraction isolated from Boscan distillate. Therefore, it is likely that methylbenzoporphyrins in addition to benzoporphyrin are present in the Shinjo sediments. a)

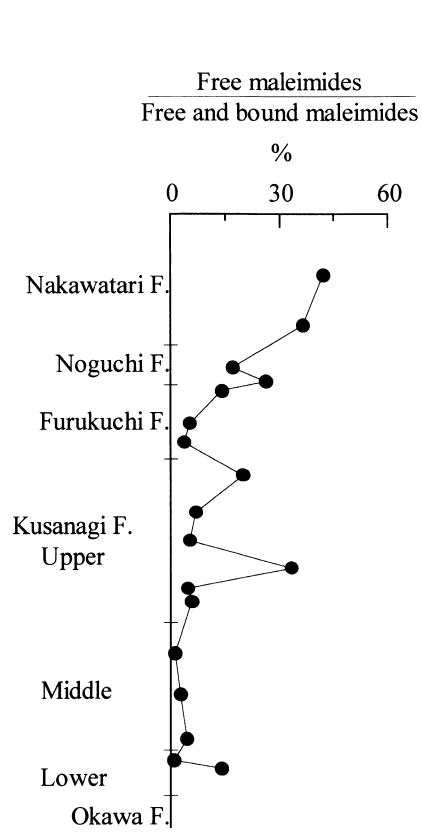

b)

c)

)
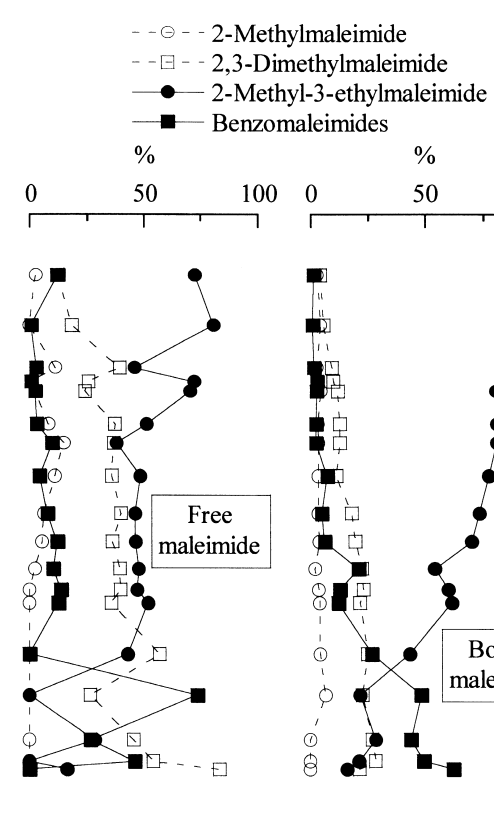

$\beta$-Methylbenzomaleimide Total methylbenzomaleimides $\%$

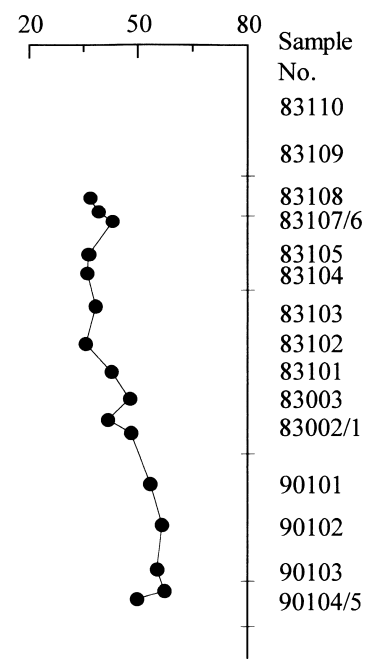

Fig. 4. Ratios of total concentrations of free maleimides to those of free and bound maleimides (a), molar compositions of free maleimides $(b)$, bound maleimides $(c)$, and ratios of $\beta$-methyl-to total methylbenzomaleimides $(d)$, $v s$. depth in the stratigraphic sequence of the Shinjo sediments. 
Diagenetic changes of maleimide ratios

The ratio of free to total maleimide concentration at each sediment sample is plotted against the depth of the sediment in Fig. 4(a). The ratio decreases from 0.44 of the top sample to 0.05 of the sediment just above the Kusanagi Formation and is almost constant over the Kusanagi Formation with some scattered values. It is remarkable that this ratio decreases in accord with increasing depth to a value near zero in the sediment region above the oil-generating zone. This phenomenon can be explained largely by the greater sensitivity of maleimide structure to diagenetic modification or decomposition, compared to the porphyrin skeleton that produces bound maleimides on oxidation.

The depth profiles of the molar compositions (in \%) of individual free maleimides are shown in Fig. 4(b), in which three of the four maleimides seem to show no dependence on the sample depth. However, the other, benzomaleimides which include methylbenzomaleimides, increased with depth in the intense oil-generating zone (middle to lower Kusanagi formation), although the data were somewhat scattered. The increasing trend of bound benzomaleimides is recognized clearly as seen in Fig. 4(c), in which benzomaleimides are more abundant than alkylmaleimides in the zone, possibly reflecting their structural stability.

The molar composition of bound 2-methyl-3ethylmaleimide, the most abundant maleimide in the upper region of this sedimentary sequence, markedly decreases with increasing of depth (Fig. 4(c)). Since 2,3-dimethylmaleimide gradually increases with increasing depth, it is possible to consider that a part of methylethyl-pyrrole or pyrroline moiety of porphyrins present in the sediments was demethylated by burial diagenesis to form dimethyl counterparts which produce bound 2,3-dimethylmaleimide on oxidation. The demethylation is proposed based on the feasibility of thermal cleavage of weak allylic bond.

It has been well known that $\beta$ - to $\alpha$-isomer ratios of methylnaphthalene or methylphenanthrene

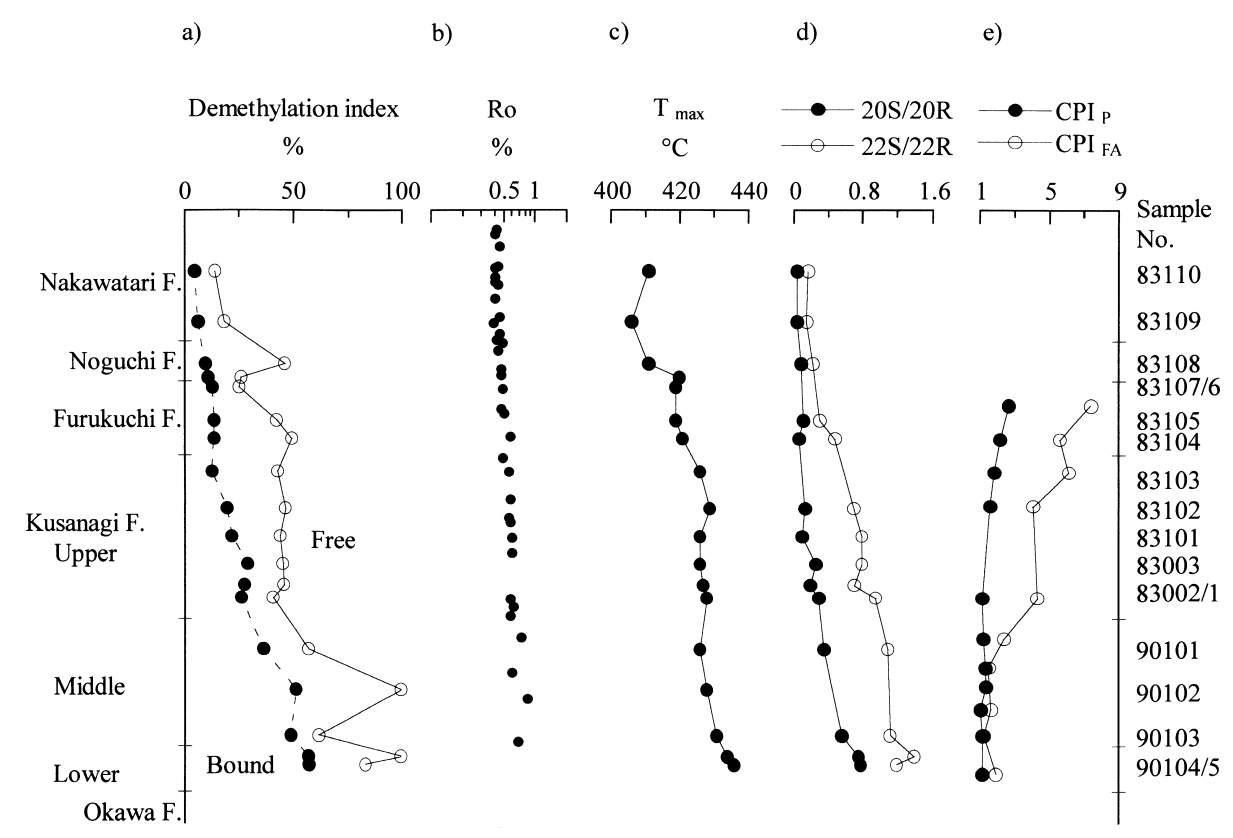

Fig. 5. Demethylation index (2,3-dimethylmaleimide/(2,3-dimethyl- + 2-ethyl-3-methylmaleimide)) (a), vitrinite reflectance (b), temperature of maximum pyrolytic yield (c), ratios of $20 S$ - to $20 R$-sterane and $22 S$ - to $22 R$-triterpane $(d)$, and Carbon Preference Indices of n-alkanes and kerogen-bound $n$-fatty acids $(e)$. The data depicted in (b)-(e) are from Taguchi et al. (1986). 
are affected by diagenesis, leading to decrease of $\alpha$-isomer because of its instability due to steric hindrance. The ratio of $\beta$-methylbenzomaleimide to the less stable $\alpha$-isomer in Fig. 4(d) increases with depth, indicating that diagenesis alters the isomer ratio of benzomaleimide.

The ratios of 2,3-dimethyl- to 2-methyl-3ethylmaleimide expressed as 2,3-dimethylmaleimide/(2,3-dimethyl- + 2-methyl-3-ethylmaleimide) with the depth of the sediments are plotted in Fig. 5(a). The ratios of the free compounds increase with depth on the whole, being steady in the upper Kusanagi Formation and scattering largely in the high EOC/TOC region. On the other hand, the ratios of bound ones show a consistently increasing trend with depth, and this depth profile agrees well with those of the other maturity parameters (Taguchi et al., 1986), such as the vitrinite reflectance (Fig. 5(b)), Rock-Eval $T_{\max }$ (Fig. 5(c)) and the ratios of 20S- to 20Rsteranes and 22S- to 22R-triterpanes (Fig. 5(d)). The carbon preference indexes (CPIs) of $n$-alkanes in the EOC and kerogen-bound fatty acids (Fig. 5(e)) decrease with depth and reach their minimum values in the intense oil-generating zone of this sequence. Therefore, the ratio of 2,3-dimethyl- to 2-methyl-3-ethylmaleimide can be used as a good demethylation index of an $n$-alkyl group like the CPI, even in the depth region where the CPIs had already reached nearly one.

The bound maleimides analyzed in this investigation were probably obtained from kerogenbound porphyrins by the oxidation treatment. If so, it is likely that the maleimide moieties in the porphyrins had largely received only burial heat with time as opposed to the free maleimides, which were affected by various additional aspects of sediment constitution. The present results suggest that bound 2-methyl-3-ethylmaleimide was affected by the burial diagenesis in such a way that corresponded to the increase in maturity of organic matter in the sediments. On the basis of these findings and interpretation, the demethylation index of bound 2-methyl-3-ethylmaleimide can be utilized as a new parameter of maturity assessment of organic matter in sediments.

\section{Conclusions}

Eighteen sediments from the Shinjo basin were analyzed for maleimides. The following conclusions were found to characterize free and bound maleimides in the Neogene sediments.

1. The sediments contained 2-methylmaleimide and its 3-n-alkyl homologs up to $\mathrm{C}_{5}$, benzomaleimide and its methyl homologs, and tetrahydrobenzomaleimide. The concentrations of free and bound maleimides varied from 0.10 to 21 and from 1.5 to $75 \mathrm{nmol} \mathrm{g}^{-1}$, respectively. In most of the sediments the predominant maleimide was 2-methyl-3-ethylmaleimide, which is likely derived from methylethyl-pyrroles or pyrrolines included in many chlorophylls and the major sedimentary porphyrins.

2. The depth profiles of both forms of maleimides were not in accordance with those of EOC and TOC, and showed a decreasing trend with increasing depth in the Kusanagi Formation, the oil-generating zone of the sequence. The concentrations of these maleimides in TOC were characteristically low in the high EOC/TOC region of the middle to lower Kusanagi Formation.

3. The ratio of total concentration of free maleimides to that of bound maleimides decreased with depth from 0.44 to 0.05 above the Kusanagi Formation and showed almost constant values near zero in the oil-generating layer, indicating greater sensitivity of free maleimide to diagenetic modification or decomposition than that of porphyrins in sediments.

4. The molar composition of bound benzomaleimides gradually increased with depth and these compounds predominated around the bottom sediments. The ratio of $\beta$-methylbenzomaleimide to the less stable $\alpha$-isomer increased with increasing depth.

5. The molar ratio of bound 2,3-dimethyl- to 2-methyl-3-ethylmaleimide increased with increasing depth and the depth profile agreed well with those of other maturity parameters.

Acknowledgments-We are grateful to Prof. J. R. Cronin, Arizona State University, who read an earlier version of this paper and provided thoughtful sugges- 
tions in addition to improved English usage. This work was supported by the Grant-in-Aid for Scientific Research (B) No. 11440166 from the Ministry of Education, Science, Sports and Culture, Japan.

\section{REFERENCES}

Baker, E. W. and Louda, J. W. (1986) Porphyrin geochemistry of Atlantic Jurrassic-Cretaceous black shales. Org. Geochem. 10, 905-914.

Barakat, A. O. and Rullkötter, J. (1998) Product distribution from oxidative degradation of sulphur-rich kerogens from the Nordlinger Ries (southern Germany). Fuel 77, 85-94.

Barakat, A. O. and Yen, T. F. (1989) The nature of porphyrins in kerogen. Evidence of entrapped etioporphyrin species. Energy and Fuels 3, 613-616.

Barwise, A. J. G. and Roberts, I. (1984) Diagenetic and catagenetic pathways for porphyrins in sediments. Org. Geochem. 6, 167-176.

Barwise, A. J. G. and Whitehead, E. V. (1980) Separation and structure of petroporphyrins. Advances in Organic Geochemistry 1979 (Maxwell, J. R. and Douglas, A. G., eds.), 181-192, Pergamon Press, Oxford.

Callot, H. J., Ocampo, R. and Albrecht, P. (1990) Sedimentary porphyrins: Correlation with biological precursors. Energy and Fuels 4, 635-639.

Chicarelli, M. I., Kaur, S. and Maxwell, J. R. (1987) Sedimentary porphyrins: Unexpected structures, occurrence and possible origins. Metal Complexes in Fossil Fuels (Filby, R. H. and Branthaver, J. F., eds.), 40-67, American Chemical Society, Washington, D.C.

Connan, J. (1974) Time-temperature relation in oil genesis. American Association of Petroleum Geologists Bulletin 58, 2516-2521.

Furlong, E. T. and Carpenter, R. (1988) Pigment preservation and remineralization in oxic coastal marine sediments. Geochim. Cosmochim. Acta 52, 87-99.

Gallango, O. and Cassani, F. (1992) Biological marker maturity parameters of marine crude oils and rock extracts from the Maracaibo Basin, Venezuela. Org. Geochem. 18, 215-224.

Grice, K., Gibbison, R., Atkinson, J. E., Schwark, L., Eckardt, C. B. and Maxwell, J. R. (1996) Maleimides (1 $\mathrm{H}$-pyrrole-2,5-diones) as molecular indicators of anoxygenic photosynthesis in ancient water columns. Geochim. Cosmochim. Acta 60, 3913-3924.

Grice, K., Schaeffer, P., Schwark, L. and Maxwell, J. R. (1997) Changes in palaeoenvironmental conditions during deposition of the Permian Kupferschiefer (Lower Rhine Basin, northwest Germany) inferred from molecular and isotopic compositions of biomarker components. Org. Geochem. 26, 677-690.

Hagiwara, M., Nomoto, S. and Shimoyama, A. (2000) Experimental simulation of thermal alteration of naphthalenes in sediments based on isomerization of methyl- and dimethylnaphthalenes. Res. Org. Geochem. 15, 43-50.

Kaneko, M., Taguchi, K. and Asakawa, T. (1983) Comments on pyrolytic analysis using ROCK-EVAL and its study case in Niigata Oil Field. Journal of Japanese Association of Petroleum Technologist 48, 319 (in Japanese).

Katsumata, H. and Shimoyama, A. (2001) Alkyl and polynuclear aromatic thiophenes in Neogene sediments of the Shinjo basin, Japan. Geochem. J. 35, 37-48.

Kaur, S., Chicarelli, I. and Maxwell, J. R. (1986) Naturally occurring benzoporphyrins: Bacterial marker pigments? J. Am. Chem. Soc. 58, 1325-1329.

Lewan, M. D. and Maynard, J. B. (1982) Factors controlling enrichment of vanadium and nickel in the bitumen of organic sedimentary rocks. Geochim. Cosmochim. Acta 46, 2547-2560.

Martin, J., Quirke, E., Shaw, G. J., Soper, P. D. and Maxwell, J. R. (1980) Petroporphyrins-II. The presence of porphyrins with extended alkyl substituents. Tetrahedron 36, 3261-3267.

Mita, H., Shigematsu, R. and Shimoyama, A. (1996) Characterization of water-extractable dicarboxylic acids in Neogene sediments of the Shinjo basin, Japan. Geochem. J. 30, 251-260.

Nomoto, S., Hagiwara, M., Nakano, Y. and Shimoyama, A. (2000) A new parameter for maturity determination of organic matter in sediments based on the claycatalyzed thermal isomerization of monomethylphenanthrenes. Bull. Chem. Soc. Jpn. 73, 1437-1443.

Ocampo, R., Bauder, C., Callot, H. J. and Albrecht, P. (1992) Porphyrins from Messel oil shale (Eocene, Germany): Structure elucidation, geochemical and biological significance, and distribution as a function of depth. Geochim. Cosmochim. Acta 56, 745761 .

Shimoyama, A. and Matsubaya, O. (1985) Stable carbon isotopes of organic matter in Neogene sediments of the Shinjo basin. Geochem. J. 19, 175-179.

Shimoyama, A., Hagishita, S. and Harada, K. (1988) Purines and pyrimidines in Neogene sediments of the Shinjo basin. Geochem. J. 22, 143-148.

Shimoyama, A., Komiya, M. and Harada, K. (1991) Low molecular weight monocarboxylic acids and $\gamma$ lactones in Neogene sediments of the Shinjo basin. Geochem. J. 25, 421-428.

Shimoyama, A., Hagiwara, M. and Nomoto, S. (2000) Naphthalenes in Neogene sediments of the Shinjo 
basin, Japan. Geochem. J. 34, 333-340.

Taguchi, K., Sasaki, K. and Usijima, N. (1970) Porphyrin pigments in the Neogene Tertiary rocks of the Shinjo oil field, Yamagata Prefecture. J. Geol. Soc. Jpn. 76, 559-566.

Taguchi, K., Shimoda, S., Itihara, Y., Imoto, N., Ishiwatari, R., Shimoyama, A., Akiyama, M. and Suzuki, N. (1986) Relationship of organic and inorganic diagenesis of Neogene Tertiary rocks, Northeastern Japan. Soc. Econ. Paleon. Miner. Sp. Publ. 38, 47-64.

Treibs, A. (1936) Chlorophyll and hemin derivatives in organic-mineral substances. Angew. Chem. 49, 682-686.

Van Berkel, G. J., Quirke, J. M. E. and Filby, R. H. (1989a) The Henryville Bed of the New Albany
shale-I. Preliminary characterization of the nickel and vanadyl porphyrins in the bitumen. Org. Geochem. 14, 119-128.

Van Berkel, G. J., Quirke, J. M. E. and Filby, R. H. (1989b) The Henryville Bed of the New Albany shale-II. Comparison of the nickel and vanadyl porphyrins in the bitumen with those generated from the kerogen during simulated catagenesis. Org. Geochem. 14, 129-144.

Verne-Mismer, J., Ocampo, R., Callot, H. J. and Albrecht, P. (1987) Isolation of a series of vanadyl tetrahydrobenzopetroporphyrins from Timahdit oil shale. Structural determination and total synthesis of the major component. J. Chem. Soc., Chem. Commun., 1581-1583. 